\title{
International Financial Networks with Intermediation: Modeling, A nalysis, and Computations
}

\author{
A nna Nagurney and J ose Cruz \\ Department of $\mathrm{F}$ inance and $\mathrm{O}$ perations $\mathrm{M}$ anagement \\ Isenberg School of Management \\ University of Massachusetts \\ A mherst, M assachusetts 01003
}

J uly 2002

\begin{abstract}
A bstract:
In this paper, we develop a framework for the modeling, analysis, and computation of solutions to international financial networks with intermediation. We consider three tiers of decision-makers consisting of: source agents in different countries, financial intermediaries, and consumers associated with the demand markets for different products in distinct currencies and countries. We model the behavior of the decision-makers, derive the equilibrium conditions, and establish the variational inequality formulation. We then utilize the variational inequality formulation to obtain qualitative properties of the equilibrium financial flow and price pattern as well as to propose an algorithm along with convergence results. Numerical examples are presented to illustrate both the model and the computational procedure. This research extends the recent results surrounding the modeling of financial networks with intermediation to the international dimension.
\end{abstract}

\section{J E L C lassification N umbers: G 15, C 6}




\section{Introduction}

Financial networks have been utilized in the study of financial systems since the work of Quesnay in 1758, who depicted the circular flow of funds in an economy as a network. Quesnay's basic idea was subsequently applied in the construction of flow of funds accounts, which are a statistical description of the flows of money and credit in an economy (cf. Board of Governors (1980), Cohen (1987), Nagurney and Hughes (1992)). However, since the flow of funds accounts are in matrix form, and, hence, two-dimensional, they fail to capture the behavior on a micro level of the various financial agents/ sectors in an economy, such as banks, households, insurance companies, etc. Moreover, as noted by the Board of Governors (1980), "the generality of the matrix tends to obscure certain structural aspects of the financial system that are of continuing interest in analysis," with the structural concepts of concern including those of financial intermediation.

Thore, in (1980), recognized some of the shortcomings of financial flow of funds accounts and developed, instead, network models of linked portfolios with financial intermediation, using decentralization/ decomposition theory. Note that, intermediation is typically associated with financial businesses, including banks, savings institutions, investment and insurance companies, etc., and the term implies borrowing for the purpose of lending, rather than for nonfinancial purposes. However, the algorithmic techniques at that time were not sufficiently advanced for the solution of general models.

Thore (1969) had earlier introduced networks, along with the mathematics, for the study of systems of linked portfolios (see also Charnes and Cooper (1967)) in the context of credit networks and made use of linear programming. Storoy, Thore, and Boyer (1975), in turn, presented a network model of the interconnection of capital markets and demonstrated how decomposition theory of mathematical programming could be exploited for the computation of equilibrium. The utility functions facing a sector were no longer restricted to being linear functions.

Nagurney, Dong, and Hughes (1992) developed a multi-sector, multi-instrument financial equilibrium model and recognized the network structure underlying the subproblems encountered in their proposed decomposition scheme, which was based on finite-dimensional variational inequality theory. Hence, financial models that were not limited to optimization 
formulations could now be handled in the more general equilibrium setting. The book by Nagurney and Siokos (1997) contains a plethora of financial network models, both singlecountry and international ones, as well as many references to the literature to that date. An overview of finance and variational inequalities can also be found in the paper by Nagurney (2001).

Nagurney and $\mathrm{Ke}$ (2001), in turn, presented a general model of financial intermediation consisting of three tiers of decision-makers, identified the network structure of the problem and studied the equilibrium price and financial flow pattern both qualitatively as well as numerically using finite-dimensional variational inequality theory. Their focus, however, was on a single country and on a single currency.

In this paper, we build upon the recent work of Nagurney and Ke (2001) and develop an international financial network model with intermediation. The development of such a model is timely for several reasons: the introduction and use of new currencies such as the euro, the proliferation of new financial products in different countries (and in different currencies), and the growth in technology which allows access to intermediaries not only in one's country.

The paper is organized as follows. In Section 2, we develop the model, describe the various decision-makers and their behaviors, and construct the equilibrium conditions, along with the variational inequality formulation. The variables are the equilibrium prices, as well as the equilibrium financial flows between the tiers of decision-makers. The model allows one to handle as many countries, source agents, financial intermediaries, as well as financial products and currencies as mandated by the particular application.

In Section 3, we derive qualitative properties of the equilibrium pattern, under appropriate assumptions, notably, the existence and uniqueness of a solution to the governing variational inequality. We also establish properties of the function that enters the variational inequality needed for proving convergence of the algorithmic scheme. In Section 4, we discuss the algorithm, which is then applied in Section 5 to several international financial network examples. We conclude the paper with Section 6 in which we summarize our results and suggest directions for future research. 


\section{The International F inancial $\mathbf{N}$ etwork Model with Intermediation}

In this Section, we develop the international financial network model with intermediation. The model consists of $L$ countries, with a typical country denoted by $l$; $I$ "source" agents in each country with sources of funds, with a typical source agent denoted by $i$, and $J$ financial intermediaries with a typical financial intermediary denoted by $j$. As mentioned in the Introduction, examples of source agents include households and businesses, whereas examples of financial intermediaries include banks, insurance companies, and investment companies, etc.

We assume that each source agent can transact with the financial intermediaries in different currencies with there being $H$ currencies in the international economy and with a typical currency being denoted by $h$. Finally, we assume that there are $K$ financial products which can be in distinct currencies and in different countries with a typical financial product (and associated with a demand market) being denoted by $k$. Hence, the financial intermediaries in the model, in addition to transacting with the source agents, also determine how to allocate the incoming financial resources among distinct uses, which are represented by the demand markets with a demand market corresponding to, for example, the market for real estate loans, household loans, or business loans, etc., which, as mentioned, can be associated with a distinct country and a distinct currency combination.

The international financial network is now described and depicted graphically in Figure 1. The top tier of nodes consists of the agents in the different countries with sources of funds, with agent $i$ in country $l$ being referred to as agent $i l$ and associated with node $i l$. There are, hence, $I L$ top-tiered nodes in the network. The middle tier of nodes consists of the financial intermediaries (which need not be country specific), with a typical intermediary $j$ associated with node $j$ in this (second) tier of nodes in the network. The bottom tier of nodes consists of the demand markets, with a typical demand market denoted for product $k$ in currency $h$ and country $l$, being associated with node $k h l$ in the bottom tier of nodes. There are, as depicted in Figure $1, J$ middle (or second) tiered nodes corresponding to the intermediaries

and $K H L$ bottom (or third) tiered nodes in the financial network. In addition, we add a node $J+1$ to the middle tier of nodes in order to represent the possible non-investment (of a portion or all of the funds) by one or more of the source agents. 


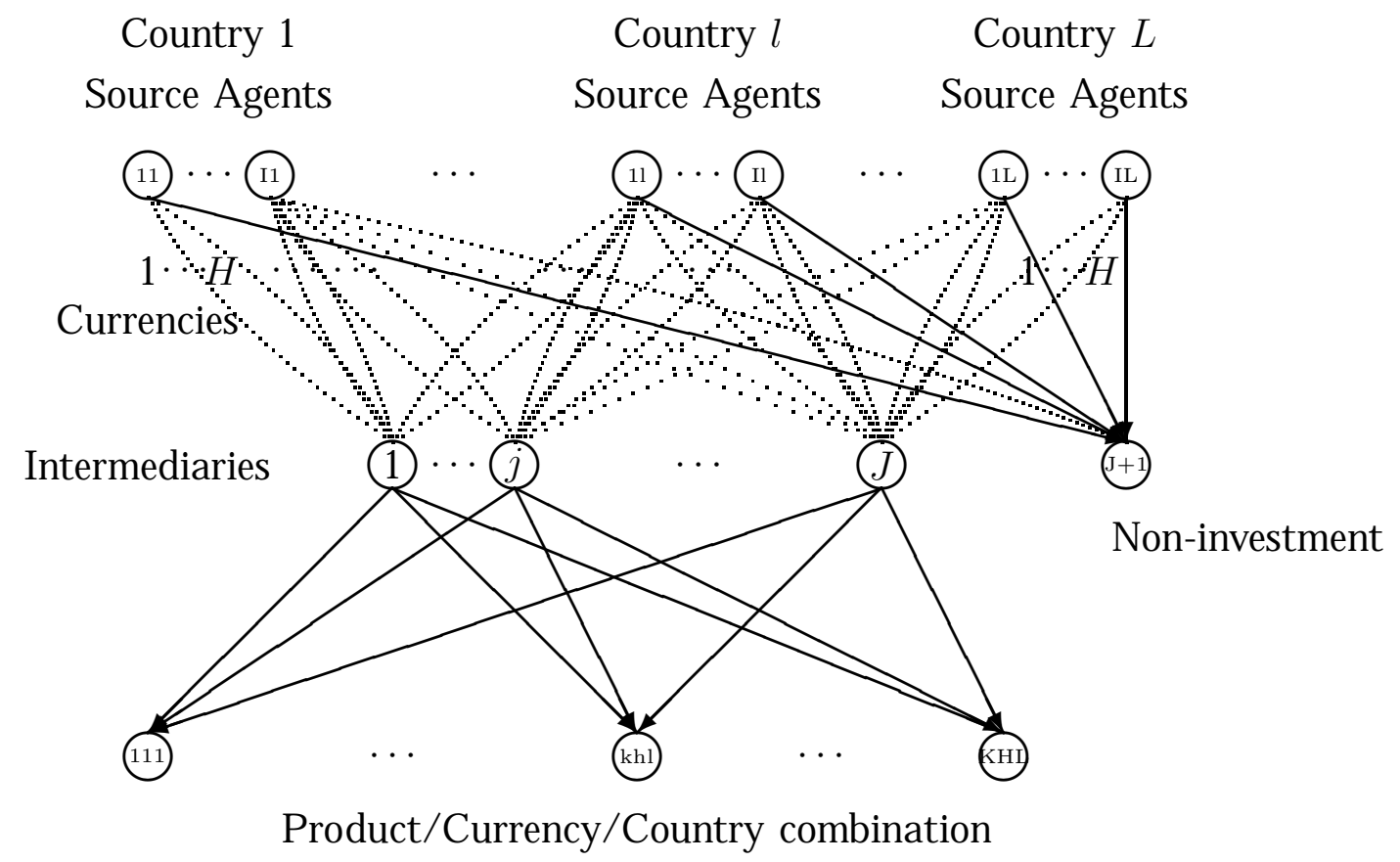

Figure 1: The Network Structure of the International Financial Economy with Intermediation and with Non-investment Allowed 
We have identified the nodes in the international financial network and now we turn to the identification of the links joining the nodes in a given tier with those in the next tier. We also associate the financial flows with the appropriate links. We assume that each agent $i$ in country $l$ has an amount of funds $S^{i l}$ in a base currency. Since we allow for $h$ currencies, there are $H$ links joining each top tier node with each middle tier node $j ; j=1, \ldots, J$. The flow on such a link $h$ joining node $i l$ with node $j$ is denoted by $x_{j h}^{i l}$ and represents the nonnegative amount invested (across all financial instruments) by agent $i$ in country $l$ in currency $h$ transacted through intermediary $j$. We group the financial flows associated with agent $i$ in country $l$ into the column vector $x^{i l} \in R_{+}^{J H}$. We then further group all such flows for all agents in all countries into the column vector $x \in R_{+}^{I L J H}$. In addition, we construct a link from each top tiered node to the second tiered node $J+1$ and associate a flow $s^{i l}$ on such a link emanating from node $i l$ to represent the possible nonnegative amount not invested by agent $i$ in country $l$.

From each intermediary node $j ; j=1, \ldots, J$, we then construct a single link to each node $k h l$ with the flow on such a link being denoted by $y_{k h l}^{j}$ and corresponding to the amount of the financial product $k$ in currency $h$ and country $l$ transacted from intermediary $j$. We group the financial flows between node $j$ and the bottom tier nodes into the column vector $y^{j} \in R_{+}^{K H L}$. All such financial flows for all the intermediaries are then further grouped into the column vector $y \in R_{+}^{J K H L}$.

Note that here we have assumed that there is a single instrument in each currency that a given source agent in a country can transact with a particular intermediary for. Of course, one could include multiple instruments in distinct currencies that could be invested in by a source agent transacting with a given intermediary pair (with a concommitant increase in notation) which would be represented by adding additional sets (as many as there are new instruments) of $H$ links between each such pair of first and second tier nodes to the financial network in Figure 1.

The notation for the prices is now given. Note that there will be prices associated with each of the tiers of nodes in the international financial network. Let $\rho_{1 j h}^{i l}$ denote a price associated with the financial instrument in currency $h$ as quoted by intermediary $j$ to agent $i l$ and we group the top tier prices into the column vector $\rho_{1} \in R_{+}^{I L J H}$. Let $\rho_{2 k h l}^{j}$, in turn, 
denote a price associated with intermediary $j$ for product $k$ in currency $h$ and country $l$ and group all such prices into the column vector $\rho_{2} \in R_{+}^{J K H L}$. Also, let $\rho_{3 k h l}$ denote a price of the financial product $k$ in currency $h$ and in country $l$, and group all such prices into the column vector $\rho_{3} \in R_{+}^{K H L}$. Finally, let $e_{h}$ denote the rate of appreciation of currency $h$ against the basic currency, which can be interpreted as the rate of return earned due to exchange rate fluctuations (see Nagurney and Siokos (1997)). These "exchange" rates are grouped into the column vector $e \in R_{+}^{H}$.

We now turn to describing the behavior of the various economic agents represented by the three tiers of nodes in Figure 1. We first focus on the top-tier agents. We then turn to the intermediaries and, subsequently, to the consumers at the demand markets.

\section{The B ehavior of the A gents with Sources of Funds and their Optimality Condi- tions}

We denote the transaction cost associated with source agent $i l$ transacting with intermediary $j$ for the instrument in currency $h$ by $c_{j h}^{i l}$ and assume that:

$$
c_{j h}^{i l}=c_{j h}^{i l}\left(x_{j h}^{i l}\right), \quad \forall i, l, j, h,
$$

that is, the cost associated with source agent $i$ in country $l$ transacting with intermediary $j$ for the instrument in currency $h$ depends on the volume of flow of the transaction. The transaction cost functions are assumed to be convex and continuously differentiable.

The total transaction costs incurred by source agent $i l$ are equal to the sum of all of his transaction costs associated with dealing with the distinct intermediaries in the different currencies. His revenue, in turn, is equal to the sum of the price (rate of return plus the rate of appreciation) that the agent can obtain for the financial instrument times the total quantity obtained/purchased of that instrument. Let now $\rho_{1 j h}^{i l *}$ denote the actual price charged agent $i l$ for the instrument in currency $h$ by intermediary $j$. Similarly, let $e_{h}^{*}$ denote the actual rate of appreciation in currency $h$. We later discuss how such prices are recovered.

We assume that each such source agent seeks to maximize net return while, simultaneously, minimizing the risk, with source agent $i l^{\prime}$ 's utility function denoted by $U^{i l}$. In 
particular, we assume, as given, a risk function for sector $i l$ and denoted by $r^{i l}$, such that

$$
r^{i l}=r^{i l}\left(x^{i l}\right), \quad \forall i, l,
$$

where $r^{i l}$ is assumed to be strictly concave and continuously differentiable. Clearly, a possible risk function could be constructed as follows. Assume a variance-covariance matrix $Q^{i l}$ associated with agent $i l$, which is of dimension $J H \times J H$, symmetric, and positive definite. Then a possible risk function for source agent $i$ in country $l$ would be given by:

$$
r^{i l}\left(x^{i l}\right)=x^{i l^{T}} Q^{i l} x^{i l}, \quad \forall i, l .
$$

In such a case, one assumes that each source agent's uncertainty, or assessment of risk, is based on a variance-covariance matrix representing the source agent's assessment of the standard deviation of the prices of the financial instruments in the distinct currencies (see also Markowitz (1959)).

We now construct the utility maximization problem facing a sector $i$ in country $l$. In particular, we can express the optimization problem facing agent $i l$ as:

$$
\text { Maximize } U^{i l}\left(x^{i l}\right)=\sum_{j=1}^{J} \sum_{h=1}^{H}\left(\rho_{1 j h}^{i l *}+e_{h}^{*}\right) x_{j h}^{i l}-\sum_{j=1}^{J} \sum_{h=1}^{H} c_{j h}^{i l}\left(x_{j h}^{i l}\right)-r^{i l}\left(x^{i l}\right) \text {, }
$$

subject to $x_{j h}^{i l} \geq 0$, for all $j, h$, and to the constraint:

$$
\sum_{j=1}^{J} \sum_{h=1}^{H} x_{j h}^{i l} \leq S^{i l}
$$

that is, the allocations of agent $i l$ 's funds among those available from the different intermediaries in distinct currencies cannot exceed his holdings. The first term in the utility function (4) denotes the revenue whereas the second term denotes the transaction costs and the last term denotes the risk. Note that the utility function given in (4) is strictly concave in the $x^{i l}$ variables. Note also that constraint (5) allows a source agent not to invest a portion (or all) of his funds, with the "slack," that is, the funds not invested by agent $i$ in country $l$ being given by $s^{i l}$. 


\section{Optimality Conditions for A II Source A gents}

The optimality conditions of all source agents $i ; i=1, \ldots, I$; in all countries: $l ; l=$ $1, \ldots, L$ (see also Bazaraa, Sherali, and Shetty (1993) and Bertsekas and T sitsiklis (1992)), under the above stated assumptions on the underlying functions, can be expressed as: determine $x^{*} \in \mathcal{K}^{1}$, satisfying

$$
\sum_{i=1}^{I} \sum_{l=1}^{L} \sum_{j=1}^{J} \sum_{h=1}^{H}\left[\frac{\partial r^{i l}\left(x^{i l *}\right)}{\partial x_{j h}^{i l}}+\frac{\partial c_{j h}^{i l}\left(x_{j h}^{i l *}\right)}{\partial x_{j h}^{i l}}-\rho_{1 j h}^{i l *}-e_{h}^{*}\right] \times\left[x_{j h}^{i l}-x_{j h}^{i l *}\right] \geq 0, \quad \forall x \in \mathcal{K}^{1},
$$

where the feasible set $\mathcal{K}^{1} \equiv\left\{x \mid x \in R_{+}^{I L J H}\right.$ and satisfies (3), $\left.\forall i, l\right\}$.

\section{The B ehavior of the Intermediaries and their Optimality Conditions}

The intermediaries (cf. Figure 1), in turn, are involved in transactions both with the source agents in the different countries, as well as with the users of the funds, that is, with the ultimate consumers associated with the markets for the distinct types of loans/ products in different currencies and countries and represented by the bottom tier of nodes of the network. Thus, an intermediary conducts transactions both with the "source" agents as well as with the consumers at the demand markets.

A n intermediary $j$ is faced with what we term a handling/ conversion cost, which may include, for example, the cost of converting the incoming financial flows into the financial loans/ products associated with the demand markets. We denote such a cost faced by intermediary $j$ by $c_{j}$ and, in the simplest case, we would have that $c_{j}$ is a function of $\sum_{i=1}^{I} \sum_{l=1}^{L} \sum_{h=1}^{H} x_{j h}^{i l}$, that is, the holding/ conversion cost of an intermediary is a function of how much he has obtained in the different currencies from the various source agents in the different countries. For the sake of generality, however, we allow the function to depend also on the amounts held by other intermediaries and, therefore, we may write:

$$
c_{j}=c_{j}(x), \quad \forall j .
$$

The intermediaries also have associated transaction costs in regards to transacting with the source agents, which we assume can be dependent on the type of currency as well as the source agent. We denote the transaction cost associated with intermediary $j$ transacting 
with agent $i l$ associated with currency $h$ by $\hat{c}_{j h}^{i l}$ and we assume that it is of the form

$$
\hat{c}_{j h}^{i l}=\hat{c}_{j h}^{i l}\left(x_{j h}^{i l}\right), \quad \forall i, l, j, h,
$$

that is, such a transaction cost is allowed to depend on the amount allocated by the particular agent to the financial instrument in a currency and transacted with the particular intermediary. In addition, we assume that an intermediary $j$ also incurs a transaction cost $c_{k h l}^{j}$ associated with transacting with demand market $k h l$, where

$$
c_{k h l}^{j}=c_{k h l}^{j}\left(y_{k h l}^{j}\right), \quad \forall j, k, h, l .
$$

Hence, the transaction costs given in (9) can vary according to the intermediary/ product/ currency/ country combination and are a function of the volume of the product transacted. We assume that the cost functions (7) - (9) are convex and continuously differentiable.

The actual price charged for the financial products by the intermediaries is denoted by $\rho_{2 k h l}^{j *}$, for intermediary $j$ and associated with transacting with consumers for product $k$ in currency $h$ and country $l$. Subsequently, we discuss how such prices are arrived at. We assume that the intermediaries are also utility maximizers with the utility functions for each being comprised of net revenue maximization as well as risk minimization.

We assume that the intermediaries have risk associated both with transacting with the various source agents in the different countries and with the consumers for the products in the different currencies and countries. Hence, we assume for each intermediary $j$ a risk function $r^{j}$, which is strictly concave in its variables and continuously differentiable, and of the form:

$$
r^{j}=r^{j}(x, y), \quad \forall j .
$$

For example, the risk for intermediary $j$ could be represented by a variance-covariance matrix denoted by $Q^{j}$ with this matrix being positive definite and of dimensions ( $I L+$ $K H L) \times(I L+K H L)$ for each intermediary $j$. Such a matrix would reflect the risk associated with transacting with the various source agents in the different countries and with the consumers at the demand markets for the products in different currencies and in different countries. If we let $x_{j}$, without any loss in generality, denote the $I L H$-dimensional column 
vector with the $i l h$-th component given by $x_{j h}^{i l}$. Indeed, then a possible risk function for intermediary $j$ could be represented by the function:

$$
r^{i l}(x, y)=\left[\begin{array}{l}
x_{j} \\
y^{j}
\end{array}\right]^{T} Q^{j}\left[\begin{array}{l}
x_{j} \\
y^{j}
\end{array}\right] .
$$

Note that, for the sake of modeling generality and flexibility, we allow the risk function for an intermediary to depend not only on the financial flows flowing "into" and "out of" that intermediary but on the other financial flows as well. The risk function given by (11) is actually a special case of the one in (10) in that it depends only on the financial volumes that the particular intermediary actually deals with.

The utility maximization problem for intermediary $j$, with his utility function expressed by $U^{j}$, and assuming net revenue maximization and risk minimization can, hence, be expressed as:

$$
\begin{aligned}
\text { Maximize } & U^{j}\left(x_{j}, y^{j}\right)=\sum_{k=1}^{k} \sum_{h=1}^{H} \sum_{l=1}^{L}\left(\rho_{2 k h l}^{j *}+e_{h}^{*}\right) x_{j h}^{i l}-c_{j}(x)-\sum_{i=1}^{I} \sum_{l=1}^{L} \sum_{h=1}^{H} \hat{c}_{j h}^{i l}\left(x_{j h}^{i l}\right) \\
- & \sum_{k=1}^{K} \sum_{h=1}^{H} \sum_{l=1}^{L} c_{k h l}^{j}\left(y_{k h l}^{j}\right)-\sum_{i=1}^{I} \sum_{l=1}^{L} \sum_{h=1}^{H}\left(\rho_{1 j h}^{i l *}+e_{h}^{*}\right) x_{j h}^{i l}-r^{j}(x, y)
\end{aligned}
$$

subject to: the nonnegativity constraints: $x_{j h}^{i l} \geq 0, y_{k h l}^{j} \geq 0$, for all $i, l, h$, and

$$
\sum_{k=1}^{K} \sum_{h=1}^{H} \sum_{l=1}^{L} y_{k h l}^{j} \leq \sum_{i=1}^{I} \sum_{l=1}^{L} \sum_{h=1}^{H} x_{j h}^{i l} .
$$

Objective function (12) expresses that the difference between the revenues (given by the first term) minus the handling cost, the two sets of transaction costs, and the payout to the source agents (given by the subsequent four terms, respectively) should be maximized, whereas the risk (see the last term in (12)) should be minimized. The utility function in (12) is concave in its variables under the above posed assumptions.

Here we assume that the financial intermediaries can compete, with the governing optimality/ equilibrium concept underlying noncooperative behavior being that of $\mathrm{N}$ ash (1950, 1951), which states that each decision-maker (intermediary) will determine his optimal 
strategies, given the optimal ones of his competitors. The optimality conditions for all financial intermediaries simultaneously, under the above stated assumptions, can be compactly expressed as (see also Gabay and M oulin (1980), Dafermos and Nagurney (1987), and Nagurney and $\mathrm{Ke}(2001))$ : determine $\left(x^{*}, y^{*}, \gamma^{*}\right) \in R_{+}^{I L J H+J K H L+J}$, such that

$$
\begin{gathered}
\sum_{j=1}^{J} \sum_{i=1}^{I} \sum_{l=}^{L} \sum_{h=1}^{H}\left[\frac{\partial r^{j}\left(x^{*}, y^{*}\right)}{\partial x_{j h}^{i l}}+\frac{\partial c_{j}\left(x^{*}\right)}{\partial x_{j h}^{i l}}+\rho_{1 j h}^{i l *}+e_{h}^{*}+\frac{\partial \hat{c}_{j h}^{i l}\left(x_{j h}^{i l *}\right)}{\partial x_{j h}^{i l}}-\gamma_{j}^{*}\right] \times\left[x_{j h}^{i l}-x_{j h}^{i l *}\right] \\
+\sum_{j=1}^{J} \sum_{k=1}^{K} \sum_{h=1}^{H} \sum_{l=1}^{L}\left[\frac{\partial r^{j}\left(x^{*}, y^{*}\right)}{\partial y_{k h l}^{j}}+\frac{\partial c_{k h l}^{j}\left(y_{k h l}^{j *}\right)}{\partial y_{k h l}^{j}}-\rho_{2 k h l}^{j *}-e_{h}^{*}+\gamma_{j}^{*}\right] \times\left[y_{k h l}^{j}-y_{k h l}^{j *}\right] \\
+\sum_{j=1}^{J}\left[\sum_{i=1}^{I} \sum_{l=1}^{L} \sum_{h=1}^{H} x_{j h}^{i l *}-\sum_{k=1}^{K} \sum_{h=1}^{H} \sum_{l=1}^{L} y_{k h l}^{j *}\right] \times\left[\gamma_{j}-\gamma_{j}^{*}\right] \geq 0, \quad \forall(x, y, \gamma) \in R_{+}^{I L J H+J K H L+J},
\end{gathered}
$$

where $\gamma_{j}$ is the Lagrange multiplier associated with constraint (9) (see Bazaraa, Sherali, and Shetty (1993)), and $\gamma$ is the $J$-dimensional column vector of Lagrange multiplers of all the intermediaries with $\gamma^{*}$ denoting the vector of optimal multipliers.

\section{The Consumers at the Demand M arkets and the Equilibrium C onditions}

We now describe the consumers located at the demand markets. The consumers take into account in making their consumption decisions not only the price charged for the financial product by the intermediaries but also their transaction costs associated with obtaining the product. Recall that there is a distinct product $\mathrm{k}$ in currency $h$ and country $l$.

We let $\hat{c}_{k h l}^{j}$ denote the transaction cost associated with obtaining product $k$ in currency $h$ in country $l$ from intermediary $j$ and recall that $y_{k l h}^{j}$ is the amount of the financial product $k$ in currency $h$ flowing between intermediary $j$ and consumers in country $l$. We assume that the transaction cost is continuous and of the general form:

$$
\hat{c}_{k h l}^{j}=\hat{c}_{k h l}^{j}(y), \quad \forall j, k, h, l .
$$

Hence, we allow for the transaction cost (from the perspective of consumers) to depend not only upon the flow of the financial product from an intermediary in the currency to the country but also on other product flows in other currencies between intermediaries and consumers in other countries. 
Denote the demand for product $k$ in currency $h$ in country $l$ by $d_{k h l}$ and assume, as given, the continuous demand functions:

$$
d_{k h l}=d_{k h l}\left(\rho_{3}\right), \quad \forall k, h, l .
$$

Thus, according to (16), the demand of consumers for the product in a currency and country depends, in general, not only on the price of the product at that demand market (and currency and country) but also on the prices of the other products at the other demand markets (and in other countries and currencies). Consequently, consumers at a demand market, in a sense, also compete with consumers at other demand markets.

The consumers take the price charged by the intermediary, which was denoted by $\rho_{2 k h l}^{j *}$ for intermediary $j$, product $k$, currency $h$, and country $l$, and the rate of appreciation in the currency, plus the transaction cost, in making their consumption decisions. The equilibrium conditions for the consumers at demand market $k h l$, thus, take the form: for all intermediaries: $j=1, \ldots, J$ :

$$
\rho_{2 k h l}^{j *}+e_{h}^{*}+\hat{c}_{k h l}^{j}\left(y^{*}\right)\left\{\begin{array}{lll}
=\rho_{3 k h l}^{*}, & \text { if } & y_{k h l}^{j *}>0 \\
\geq \rho_{3 k h l}^{*}, & \text { if } & y_{k h l}^{j *}=0,
\end{array}\right.
$$

and

$$
d_{k h l}\left(\rho_{3}^{*}\right)\left\{\begin{array}{lll}
=\sum_{j=1}^{J} y_{k h l}^{j *}, & \text { if } & \rho_{3 k h l}^{*}>0 \\
\leq \sum_{j=1}^{J} y_{k h l}^{j *}, & \text { if } & \rho_{3 k h l}^{*}=0 .
\end{array}\right.
$$

Conditions (17) state that consumers at demand market $k h l$ will purchase the product from intermediary $j$, if the price charged by the intermediary for the product and the appreciation rate for the currency plus the transaction cost (from the perspective of the consumer) does not exceed the price that the consumers are willing to pay for the product in that currency and country, i.e., $\rho_{3 k h l}^{*}$. Note that, according to (17), if the transaction costs are identically equal to zero, then the price faced by the consumers for a given product is the price charged by the intermediary for the particular product and currency in the country plus the rate of appreciation in the currency.

Condition (18), on the other hand, states that, if the price the consumers are willing to pay for the financial product at a demand market is positive, then the quantity of the product 
transacted by the intermediaries with the consumers at the demand market is precisely equal to the demand. Hence, the prices are the market clearing prices for the distinct financial products.

Observe that, in the context of consumption decisions in the form of the ultimate uses of funds, we have utilized demand functions, rather than utility functions, as was the case for the source agents and the intermediaries, who were assumed to be faced with utility functions composed of a net revenue term to be maximized and a risk term to be minimized. We expect the number of consumers to be much greater than that of the source agents and the intermediaries and, thus, believe that the above formulation is the more natural and tractable one.

In equilibrium, conditions (17) and (18) will have to hold for all intermediaries and for all demand markets and these, in turn, can be expressed also as an inequality analogous to those in (6) and (14) and given by: determine $\left(y^{*}, \rho_{3}^{*}\right) \in R_{+}^{(J+1) K H L}$, such that

$$
\begin{gathered}
\sum_{j=1}^{J} \sum_{k=1}^{K} \sum_{h=1}^{H} \sum_{l=1}^{L}\left[\rho_{2 k h l}^{j *}+e_{h}^{*}+\hat{c}_{k h l}^{j}\left(y^{*}\right)-\rho_{3 k h l}^{*}\right] \times\left[y_{k h l}^{j}-y_{k h l}^{j *}\right] \\
+\sum_{k=1}^{K} \sum_{h=1}^{H} \sum_{l=1}^{L}\left[\sum_{j=1}^{J} y_{k h l}^{j *}-d_{k h l}\left(\rho_{3}^{*}\right)\right] \times\left[\rho_{3 k h l}-\rho_{3 k h l}^{*}\right] \geq 0, \quad \forall\left(y, \rho_{3}\right) \in R_{+}^{(J+1) K H L} .
\end{gathered}
$$

\section{The Equilibrium C onditions for the International Financial E conomy}

In equilibrium, the financial flows that the source agents in different countries transact with the intermediaries must coincide with those that the intermediaries actually accept from them. In addition, the amounts of the financial products that are obtained by the consumers in the different countries and currencies must be equal to the amounts that the intermediaries actually provide. Hence, although there may be competition between decision-makers at the same level of tier of nodes of the financial network there must be, in a sense, cooperation between decision-makers associated with pairs of nodes (through positive flows on the links joining them). Thus, in equilibrium, the prices and financial flows must satisfy the sum of the optimality conditions (6) and (14) and the equilibrium conditions (19). We make these relationships rigorous through the subsequent definition and variational inequality derivation below. 


\section{Definition 1: International Financial $\mathbf{N}$ etwork Equilibrium with Intermediation}

The equilibrium state of the international financial network with intermediation is one where the financial flows between the tiers of the network coincide and the financial flows and prices satisfy the sum of conditions (6), (14), and (19).

The equilibrium state is equivalent to the following:

\section{Theorem 1: Variational Inequality Formulation}

The equilibrium conditions governing the international financial network with inter mediation according to Definition 1 are equivalent to the solution of the variational inequality given by: determine $\left(x^{*}, y^{*}, \gamma^{*}, \rho_{3}^{*}\right) \in \mathcal{K}$, satisfying:

$$
\begin{gathered}
\sum_{i=1}^{I} \sum_{l=1}^{L} \sum_{j=1}^{J} \sum_{h=1}^{H}\left[\frac{\partial r^{i l}\left(x^{i l *}\right)}{\partial x_{j h}^{i l}}+\frac{\partial r^{j}\left(x^{*}, y^{*}\right)}{\partial x_{j h}^{i l}}+\frac{\partial c_{j h}^{i l}\left(x_{j h}^{i l *}\right)}{\partial x_{j h}^{i l}}+\frac{\partial c_{j}\left(x^{*}\right)}{\partial x_{j h}^{i l}}+\frac{\partial \hat{c}_{j h}^{i l}\left(x_{j h}^{i l *}\right)}{\partial x_{j h}^{i l}}-\gamma_{j}^{*}\right] \times\left[x_{j h}^{i l}-x_{j h}^{i l *}\right] \\
+\sum_{j=1}^{J} \sum_{k=1}^{K} \sum_{h=1}^{H} \sum_{l=1}^{L}\left[\frac{\partial r^{j}\left(x^{*}, y^{*}\right)}{\partial y_{k h l}^{j}}+\frac{\partial c_{k h l}^{j}\left(y_{k h l}^{j *}\right)}{\partial y_{k h l}^{j}}+\gamma_{j}^{*}+\hat{c}_{k h l}^{j}\left(y^{*}\right)-\rho_{3 k h l}^{*}\right] \times\left[y_{k h l}^{j}-y_{k h l}^{j *}\right] \\
+\sum_{j=1}^{J}\left[\sum_{i=1}^{I} \sum_{l=1}^{L} \sum_{h=1}^{H} x_{j h}^{i l *}-\sum_{k=1}^{K} \sum_{h=1}^{H} \sum_{l=1}^{L} y_{k h l}^{j *}\right] \times\left[\gamma_{j}-\gamma_{j}^{*}\right] \\
+\sum_{k=1}^{K} \sum_{h=1}^{H} \sum_{l=1}^{L}\left[\sum_{j=1}^{J} y_{k h l}^{j *}-d_{k h l}\left(\rho_{3}^{*}\right)\right] \times\left[\rho_{3 k h l}-\rho_{3 k h l}^{*}\right] \geq 0, \quad \forall\left(x, y, \gamma, \rho_{3}\right) \in \mathcal{K},
\end{gathered}
$$

where $\mathcal{K} \equiv\left\{\mathcal{K}^{1} \times \mathcal{K}^{2}\right\}$, where $\mathcal{K}^{2} \equiv\left\{\left(y, \gamma, \rho_{3}\right) \mid\left(y, \gamma, \rho_{3}\right) \in R_{+}^{J K H L+J+K H L}\right\}$.

\section{Proof:}

We first establish that the equilibrium conditions imply variational inequality (20). Indeed, summation of inequalities (6), (14), and (19), after algebraic simplifications, yields variational inequality (20).

We now establish the converse, that is, that a solution to variational inequality (20) satisfies the sum of conditions (6), (14), and (19), and is, hence, an equilibrium.

To inequality (20), add the term: $-\rho_{1 j h}^{i l *}-e_{h}^{*}+\rho_{1 j h}^{i l *}+e_{h}^{*}$ to the term in the first set of brackets (preceding the first multiplication sign). Similarly, add the term: $-\rho_{2 k h l}^{j *}-e_{h}^{*}+\rho_{2 k h l}^{j *}+e_{h}^{*}$ to 
the term in brackets preceding the second multiplication sign in (20). The addition of such terms does not change (20) since the value of these terms is zero and yields:

$$
\begin{gathered}
\sum_{i=1}^{I} \sum_{l=1}^{L} \sum_{j=1}^{J} \sum_{h=1}^{H}\left[\frac{\partial r^{i l}\left(x^{i l *}\right)}{\partial x_{j h}^{i l}}+\frac{\partial r^{j}\left(x^{*}, y^{*}\right)}{\partial x_{j h}^{i l}}+\frac{\partial c_{j h}^{i l}\left(x_{j h}^{i l *}\right)}{\partial x_{j h}^{i l}}+\frac{\partial c_{j}\left(x^{*}\right)}{\partial x_{j h}^{i l}}+\frac{\partial \hat{c}_{j h}^{i l}\left(x_{j h}^{i l *}\right)}{\partial x_{j h}^{i l}}-\gamma_{j}^{*}-\rho_{1 j h}^{i l *}-e_{h}^{*}+\rho_{1 j h}^{i l *}+e_{h}^{*}\right] \\
+\sum_{j=1}^{J} \sum_{k=1}^{K} \sum_{h=1}^{H} \sum_{l=1}^{L}\left[\frac{\partial r_{j h}^{i l}-x_{j h}^{i l *}\left(x^{*}, y^{*}\right)}{\partial y_{k h l}^{j}}+\frac{\partial c_{k h l}^{j}\left(y_{k h l}^{j *}\right)}{\partial y_{k h l}^{j}}+\gamma_{j}^{*}+\hat{c}_{k h l}^{j}\left(y^{*}\right)-\rho_{3 k h l}^{*}-\rho_{2 k h l}^{j *}-e_{h}^{*}+\rho_{2 k h l}^{j *}+e_{h}^{*}\right] \\
\times\left[y_{k h l}^{j}-y_{k h l}^{j *}\right] \\
+\sum_{j=1}^{J}\left[\sum_{i=1}^{I} \sum_{l=1}^{L} \sum_{h=1}^{H} x_{j h}^{i l *}-\sum_{k=1}^{K} \sum_{h=1}^{H} \sum_{l=1}^{L} y_{k h l}^{j *}\right] \times\left[\gamma_{j}-\gamma_{j}^{*}\right] \\
+\sum_{k=1}^{K} \sum_{h=1}^{H} \sum_{l=1}^{L}\left[\sum_{j=1}^{J} y_{k h l}^{j *}-d_{k h l}\left(\rho_{3}^{*}\right)\right] \times\left[\rho_{3 k h l}-\rho_{3 k h l}^{*}\right] \geq 0, \quad \forall\left(x, y, \gamma, \rho_{3}\right) \in \mathcal{K},
\end{gathered}
$$

which, in turn, can be rewritten as:

$$
\begin{gathered}
\sum_{i=1}^{I} \sum_{l=1}^{L} \sum_{j=1}^{J} \sum_{h=1}^{H}\left[\frac{\partial r^{i l}\left(x^{i l *}\right)}{\partial x_{j h}^{i l}}+\frac{\partial c_{j h}^{i l}\left(x_{j h}^{i l *}\right)}{\partial x_{j h}^{i l}}-\rho_{1 j h}^{i l *}-e_{h}^{*}\right] \times\left[x_{j h}^{i l}-x_{j h}^{i l *}\right] \\
+\sum_{j=1}^{J} \sum_{i=1}^{I} \sum_{l=}^{L} \sum_{h=1}^{H}\left[\frac{\partial r^{j}\left(x^{*}, y^{*}\right)}{\partial x_{j h}^{i l}}+\frac{\partial c_{j}\left(x^{*}\right)}{\partial x_{j h}^{i l}}+\rho_{1 j h}^{i l *}+e_{h}^{*}+\frac{\partial \hat{c}_{j h}^{i l}\left(x_{j h}^{i l *}\right)}{\partial x_{j h}^{i l}}-\gamma_{j}^{*}\right] \times\left[x_{j h}^{i l}-x_{j h}^{i l *}\right] \\
+\sum_{j=1}^{J} \sum_{k=1}^{K} \sum_{h=1}^{H} \sum_{l=1}^{L}\left[\frac{\partial r^{j}\left(x^{*}, y^{*}\right)}{\partial y_{k h l}^{j}}+\frac{\partial c_{k h l}^{j}\left(y_{k h l}^{j *}\right)}{\partial y_{k h l}^{j}}-\rho_{2 k h l}^{j *}-e_{h}^{*}+\gamma_{j}^{*}\right] \times\left[y_{k h l}^{j}-y_{k h l}^{j *}\right] \\
+\sum_{j=1}^{J} \sum_{k=1}^{K} \sum_{h=1}^{H} \sum_{l=1}^{L}\left[\rho_{2 k h l}^{j *}+e_{h}^{*}+\hat{c}_{k h l}^{j}\left(y^{*}\right)-\rho_{3 k h l}^{*}\right] \times\left[y_{k h l}^{j}-y_{k h l}^{j *}\right] \\
+\sum_{j=1}^{J}\left[\sum_{i=1}^{I} \sum_{l=1}^{L} \sum_{h=1}^{H} x_{j h}^{i l *}-\sum_{k=1}^{K} \sum_{h=1}^{H} \sum_{l=1}^{L} y_{k h l}^{j *}\right] \times\left[\gamma_{j}-\gamma_{j}^{*}\right] \\
+\sum_{k=1}^{K} \sum_{h=1}^{H} \sum_{l=1}^{L}\left[\sum_{j=1}^{J} y_{k h l}^{j *}-d_{k h l}\left(\rho_{3}^{*}\right)\right] \times\left[\rho_{3 k h l}-\rho_{3 k h l}^{*}\right] \geq 0 .
\end{gathered}
$$

But inequality (22) is equivalent to the sum of conditions (6), (14), and (19), and hence that financial flow and price pattern is an equilibrium according to Definition 1. 
We now put variational inequality (20) into standard form which will be utilized in the subsequent sections. For additional background on variational inequalities and their applications, see the book by Nagurney (1999). In paricular, we have that variational inequality (20) can be expressed as:

$$
\left\langle F\left(X^{*}\right)^{T}, X-X^{*}\right\rangle \geq 0, \quad \forall X \in \mathcal{K},
$$

where $X \equiv\left(x, y, \gamma, \rho_{3}\right)$ and $F(X) \equiv\left(F_{i l j h}, F_{j k h l}, F_{j}, F_{k h l}\right)_{i=1, \ldots, I ; l=1, \ldots, L ; j=1, \ldots, J ; h=1, \ldots, H}$, and the specific components of $F$ are given by the functional terms preceding the multiplication signs in (20), respectively. The term $\langle\cdot, \cdot\rangle$ denotes the inner product in $N$-dimensional Euclidean space.

We now describe how to recover the prices associated with the first two tiers of nodes in the international financial network. Clearly, the components of the vector $\rho_{3}^{*}$ are obtained directly from the solution of variational inequality (20) as will be demonstrated explicitly through several numerical examples in Section 5 . In order to recover the second tier prices associated with the intermediaries and the exchange rates one can (after solving variational inequality (20) for the particular numerical problem) either (cf. (17)) set $\rho_{2 k h l}^{j *}+e_{h}^{*}=$ $\rho_{3 k h l}^{*}-\hat{c}_{k h l}\left(y^{*}\right)$, for any $j, k, h, l$ such that $y_{k h l}^{j *}>0$, or (cf. (14)) for any $y_{k h l}^{j *}>0$, set $\rho_{2 k h l}^{j *}+e_{h}^{*}=\frac{\partial r^{j}\left(x^{*}, y^{*}\right)}{\partial y_{k h l}^{j}}+\frac{\partial c_{k h l}^{j}\left(y_{k h l}^{j *}\right)}{\partial y_{k h l}^{j}}-\gamma_{j}^{*}$.

Similarly, from (14) we can infer that the top tier prices comprising the vector $\rho_{1}^{*}$ can be recovered (once the variational inequality (20) is solved with particular data) thus: for any $i, l, j, h$, such that $x_{j h}^{i l *}>0$, set $\rho_{1 j h}^{i l *}+e_{h}^{*}=\gamma_{j}^{*}-\frac{\partial r^{j}\left(x^{*}, y^{*}\right)}{\partial x_{j h}^{l}}-\frac{\partial c_{j}\left(x^{*}\right)}{\partial x_{j h}^{i l}}-\frac{\partial \hat{c}_{h h}^{i l}\left(x_{j h}^{i l *}\right)}{\partial x_{j h}^{i l}}$. 


\section{Q ualitative Properties}

In this Section, we provide some qualitative properties of the solution to variational inequality (20). In particular, we derive existence and uniqueness results. We also investigate properties of the function $F$ (cf. (23)) that enters the variational inequality of interest here.

Since the feasible set is not compact we cannot derive existence simply from the assumption of continuity of the functions. Nevertheless, we can impose a rather weak condition to guarantee existence of a solution pattern. Let

$$
\mathcal{K}_{b}=\left\{\left(x, y, \gamma, \rho_{3}\right) \mid 0 \leq x \leq b_{1} ; 0 \leq y \leq b_{2} ; 0 \leq \gamma \leq b_{3} ; 0 \leq \rho_{3} \leq b_{4}\right\}
$$

where $b=\left(b_{1}, b_{2}, b_{3}, b_{4}\right) \geq 0$ and $x \leq b_{1} ; y \leq b_{2} ; \gamma \leq b_{3} ; \rho_{3} \leq b_{4}$ means that $x_{j h}^{i l} \leq b_{1}$; $y_{k h l}^{j} \leq b_{2} ; \gamma_{j} \leq b_{3} ;$ and $\rho_{3 k h l} \leq b_{4}$ for all $i, l, j, k, h$. Then $\mathcal{K}_{b}$ is a bounded closed convex subset of $R^{I L J H+J K H L+J+K H L}$. Thus, the following variational inequality

$$
\left\langle F\left(X^{b}\right)^{T}, X-X^{b}\right\rangle \geq 0, \quad \forall X^{b} \in \mathcal{K}_{b},
$$

admits at least one solution $X^{b} \in \mathcal{K}_{b}$, from the standard theory of variational inequalities, since $\mathcal{K}_{b}$ is compact and $F$ is continuous. Following Kinderlehrer and Stampacchia (1980) (see also Theorem 1.5 in Nagurney (1999)), we then have:

\section{Theorem 2}

Variational inequality (20) admits a solution if and only if there exists a $b>0$, such that variational inequality (25) admits a solution in $\mathcal{K}_{b}$ with

$$
x^{b}<b_{1}, \quad y^{b}<b_{2}, \quad \gamma^{b}<b_{3}, \quad \rho_{3}^{b}<b_{4} .
$$

\section{Theorem 3: Existence}

Suppose that there exist positive constants $M, N, R$, with $R>0$, such that:

$$
\frac{\partial r^{i l}\left(x^{i l *}\right)}{\partial x_{j h}^{i l}}+\frac{\partial r^{j}\left(x^{*}, y^{*}\right)}{\partial x_{j h}^{i l}}+\frac{\partial c_{j h}^{i l}\left(x_{j h}^{i l *}\right)}{\partial x_{j h}^{i l}}+\frac{\partial c_{j}\left(x^{*}\right)}{\partial x_{j h}^{i l}}+\frac{\partial \hat{c}_{j h}^{i l}\left(x_{j h}^{i l *}\right)}{\partial x_{j h}^{i l}} \geq M, \forall x \text { with } x_{j h}^{i l} \geq N, \forall i, l, j, h \text {, }
$$




$$
\begin{gathered}
\frac{\partial r^{j}\left(x^{*}, y^{*}\right)}{\partial y_{k h l}^{j}}+\frac{\partial c_{k h l}^{j}\left(y_{k h l}^{j *}\right)}{\partial y_{k h l}^{j}}+\hat{c}_{k h l}^{j}\left(y^{*}\right) \geq M, \quad \forall y \text { with } y_{k h l}^{j} \geq N, \quad \forall j, k, h, l, \\
d_{k}\left(\rho_{3}^{*}\right) \leq N, \quad \forall \rho_{3} \text { with } \rho_{3 k h l}>R, \quad \forall k, h, l .
\end{gathered}
$$

Then variational inequality (20); equivalently, variational inequality (23), admits at least one solution.

Proof: Follows using analogous arguments as the proof of existence for Proposition 1 in Nagurney and Zhao (1993) (see also the existence proof in Nagurney and Ke (2001)).

A ssumptions (27) and (28) are reasonable from an economics perspective, since when the financial flow between a source agent and intermediary or an intermediary and demand market is large, we can expect the corresponding sum of the associated marginal risks and marginal costs of transaction and handling to exceed a positive lower bound. Moreover, in the case where the demand price of the financial product in a currency and country as perceived by consumers at a demand market is high, we can expect that the demand for the financial product at the demand market to not exceed a positive bound.

We now establish additional qualitative properties both of the function $F$ that enters the variational inequality problem (cf. (20) and (23)), as well as uniqueness of the equilibrium pattern. Monotonicity and Lipschitz continuity of $F$ will be utilized in Section 4 to establish convergence of the proposed algorithmic scheme. Since the proofs of Theorems 4 and 5 below are similar to the analogous proofs in Nagurney and Ke (2001) they are omitted here. Additional background on the properties establish below can be found in the books by Nagurney and Siokos (1997) and Nagurney (1999).

\section{Theorem 4: M onotonicity}

Suppose that the risk function $r^{i l} ; i=1, \ldots, I ; l=1, \ldots, L$, and $r^{j} ; j=1, \ldots, J$, are strictly convex and that the $c_{j h}^{i l}, c_{j}, \hat{c}_{j h}^{i l}$, and $c_{k h l}^{j}$ functions are convex; the $\hat{c}_{k h l}^{j}$ functions are monotone increasing, and the $d_{k}$ functions are monotone decreasing functions, for all $i, l, j, h, k$. Then the vector function $F$ that enters the variational inequality (20) is monotone, that is,

$$
\left\langle\left(F\left(X^{\prime}\right)-F\left(X^{\prime \prime}\right)\right)^{T}, X^{\prime}-X^{\prime \prime}\right\rangle \geq 0, \quad \forall X^{\prime}, X^{\prime \prime} \in \mathcal{K} .
$$


Monotonicity plays a role in the qualitative analysis of variational inequality problems similar to that played by convexity in the context of optimization problems.

\section{Theorem 5: Strict M onotonicity}

A ssume all the conditions of Theorem 4. In addition, suppose that one of the families of convex functions $c_{j h}^{i l} ; i=1, \ldots, I ; l=1, \ldots, L ; j=1, \ldots, J ; h=1, \ldots, H, c_{j} ; j=1, \ldots, J ; \hat{c}_{j h}^{i l}$; $i=1, \ldots, I ; l=1, \ldots, L ; j=1, \ldots, J ; h=1, \ldots, H$, and $c_{k h l}^{j} ; j=1, \ldots, J ; k=1, \ldots, K$; $h=1, \ldots, H$, and $l=1, \ldots, L$, is a family of strictly convex functions. Suppose also that $\hat{c}_{k h l}^{j} ; j=1, \ldots, J ; k=1, \ldots, K ; h=1, \ldots, H ; l=1, \ldots, L$, and $-d_{k} ; k=1, \ldots, K$, are strictly monotone. Then, the vector function $F$ that enters the variational inequality (20) is strictly monotone, with respect to $\left(x, y, \rho_{3}\right)$, that is, for any two $X^{\prime}, X^{\prime \prime}$ with $\left(x^{\prime}, y^{\prime}, \rho_{3}^{\prime}\right) \neq\left(x^{\prime \prime}, y^{\prime \prime}, \rho_{3}{ }^{\prime \prime}\right)$

$$
\left\langle\left(F\left(X^{\prime}\right)-F\left(X^{\prime \prime}\right)\right)^{T}, X^{\prime}-X^{\prime \prime}\right\rangle>0 .
$$

\section{Theorem 6: U niqueness}

A ssuming the conditions of Theorem 5 , there must be a unique financial flow pattern $\left(x^{*}, y^{*}\right)$, and a unique demand price price vector $\rho_{3}^{*}$ satisfying the equilibrium conditions of the international financial network with intermerdiation. In other words, if the variational inequality (20) admits a solution, then that is the only solution in $\left(x, y, \rho_{3}\right)$.

Proof: Under the strict monotonicity result of Theorem 5, uniqueness follows from the standard variational inequality theory (cf. K inderlehrer and Stampacchia (1980)) 


\section{Theorem 7: Lipschitz Continuity}

The function that enters the variational inequality problem (20) is Lipschitz continuous, that is,

$$
\left\|F\left(X^{\prime}\right)-F\left(X^{\prime \prime}\right)\right\| \leq L\left\|X^{\prime}-X^{\prime \prime}\right\|, \quad \forall X^{\prime}, X^{\prime \prime} \in \mathcal{K} \text {, where } L>0,
$$

under the following conditions:

(i). $r^{i l}, r^{j}, c_{j h}^{i l}, c_{j}, \hat{c}_{j h}^{i l}, c_{k h l}^{j}$ have bounded second-order derivatives, for all $i, l, j, h, k$;

(ii). $\hat{c}_{k h l}^{j}$, and $d_{k}$ have bounded first-order derivatives for all $j, k, h, l$.

Proof: The result is direct by applying a mid-value theorem from calculus to the vector function $F$ that enters the variational inequality problem (20).

It is worth noting that the risk functions of the form (3) and (11) have bounded secondorder derivatives.

In the next Section, we will utilize the conditions of monotonicity and Lipschitz continuity in order to establish the convergence of the algorithm for the solution of the equilibrium financial flows and prices satisfying variational inequality (20). 


\section{The A Igorithm}

In this Section, we consider the computation of solutions to variational inequality (20). The algorithm that we propose is the modified projection method of Korpelevich (1977), which is guaranteed to solve any variational inequality problem in standard form (see (23)) provided that certain conditions are satisfied by the function $F$ that enters the variational inequality problem and that a solution exists. The realization of the modified projection method for the variational inequality (20) (for further details see also Nagurney and Siokos (1997)) is as follows, where $\mathcal{T}$ denotes an iteration counter:

\section{M odified Projection M ethod for the Solution of Variational Inequality (20)}

\section{Step 0: Initialization}

Set $\left(x^{0}, y^{0}, \gamma^{0}, \rho_{3}^{0}\right) \in \mathcal{K}$. Let $\mathcal{T}=1$ and set $\alpha$ such that $0<\alpha \leq \frac{1}{L}$, where $L$ is the Lipschitz constant for the problem.

\section{Step 1: Computation}

Compute $\left(\bar{x}^{\mathcal{T}}, \bar{y}^{\mathcal{T}}, \bar{\gamma}^{\mathcal{T}}, \bar{\rho}_{3}{ }^{\mathcal{T}}\right) \in \mathcal{K}$ by solving the variational inequality subproblem:

$$
\begin{gathered}
\sum_{i=1}^{I} \sum_{l=1}^{L} \sum_{j=1}^{J} \sum_{h=1}^{H}\left[\bar{x}_{j h}^{i l \mathcal{T}}+\alpha\left(\frac{\partial r^{i l}\left(\bar{x}^{i l \mathcal{T}-1}\right)}{\partial x_{j h}^{i l}}+\frac{\partial r^{j}\left(\bar{x}^{\mathcal{T}-1}, \bar{y}^{\mathcal{T}-1}\right)}{\partial x_{j h}^{i l}}+\frac{\partial c_{j h}^{i l}\left(\bar{x}_{j h}^{i l} \mathcal{T}-1\right)}{\partial x_{j h}^{i l}}+\frac{\partial c_{j}\left(\bar{x}^{\mathcal{T}-1}\right)}{\partial x_{j h}^{i l}}+\frac{\partial \hat{c}_{j h}^{i l}\left(\bar{x}_{j h}^{i l \mathcal{T}-1}\right)}{\partial x_{j h}^{i l}}\right.\right. \\
\left.\left.+\bar{\gamma}_{j}^{\mathcal{T}-1}\right)-\bar{x}_{j h}^{i l \mathcal{T}-1}\right] \times\left[x_{j h}^{i l}-\bar{x}_{j h}^{i l} \mathcal{T}\right] \\
+\sum_{j=1}^{J} \sum_{k=1}^{K} \sum_{h=1}^{H} \sum_{l=1}^{L}\left[\bar{y}_{k h l}^{j \mathcal{T}}+\alpha\left(\frac{\partial r^{j}\left(\bar{x}^{\mathcal{T}-1}, \bar{y}^{\mathcal{T}-1}\right)}{\partial y_{k h l}^{j}}+\frac{\partial c_{k h l}^{j}\left(\bar{y}_{k h l}^{j \mathcal{T}-1}\right)}{\partial y_{k h l}^{j}}+\bar{\gamma}_{j}^{\mathcal{T}-1}+\hat{c}_{k h l}^{j}\left(\bar{y}^{\mathcal{T}-1}\right)-\bar{\rho}_{3 k h l}^{\mathcal{T}-1}\right)-\bar{y}_{k h l}^{j \mathcal{T}-1}\right] \\
+\sum_{j=1}^{J}\left[\bar{\gamma}_{k h l}^{j}-\bar{y}_{k h l}^{j \mathcal{T}}+\alpha\left(\sum_{i=1}^{I} \sum_{l=1}^{L} \sum_{h=1}^{H} \bar{x}_{j h}^{i l \mathcal{T}-1}-\sum_{k=1}^{K} \sum_{h=1}^{H} \sum_{l=1}^{L} \bar{y}_{k h l}^{j \mathcal{T}-1}\right)-\bar{\gamma}_{j}^{\mathcal{T}-1}\right] \times\left[\gamma_{j}-\bar{\gamma}_{j}^{\mathcal{T}}\right] \\
+\sum_{k=1}^{K} \sum_{h=1}^{H} \sum_{l=1}^{L}\left[\bar{\rho}_{3 k h l}^{\mathcal{T}}+\alpha\left(\sum_{j=1}^{J} \bar{y}_{k h l}^{j \mathcal{T}-1}-d_{k h l}\left(\bar{\rho}_{3}^{\mathcal{T}-1}\right)\right)-\bar{\rho}_{3 k h l}^{\mathcal{T}-1}\right] \times\left[\rho_{3 k h l}-\bar{\rho}_{3 k h l}^{\mathcal{T}}\right] \geq 0 \\
\forall\left(x, y, \gamma, \rho_{3}\right) \in \mathcal{K} .
\end{gathered}
$$




\section{Step 2: A daptation}

Compute $\left(x^{\mathcal{T}}, y^{\mathcal{T}}, \gamma^{\mathcal{T}}, \rho_{3}^{\mathcal{T}}\right) \in \mathcal{K}$ by solving the variational inequality subproblem:

$$
\begin{gathered}
\sum_{i=1}^{I} \sum_{l=1}^{L} \sum_{j=1}^{J} \sum_{h=1}^{H}\left[x_{j h}^{i l \mathcal{T}}+\alpha\left(\frac{\partial r^{i l}\left(\bar{x}^{i l \mathcal{T}}\right)}{\partial x_{j h}^{i l}}+\frac{\partial r^{j}\left(\bar{x}^{\mathcal{T}}, \bar{y}^{\mathcal{T}}\right)}{\partial x_{j h}^{i l}}+\frac{\partial c_{j h}^{i l}\left(\bar{x}_{j h}^{i l \mathcal{T}}\right)}{\partial x_{j h}^{i l}}+\frac{\partial c_{j}\left(\bar{x}^{\mathcal{T}}\right)}{\partial x_{j h}^{i l}}+\frac{\partial \hat{c}_{j h}^{i l}\left(\bar{x}_{j h}^{i l \mathcal{T}}\right)}{\partial x_{j h}^{i l}}\right.\right. \\
\left.\left.-\bar{\gamma}_{j}^{\mathcal{T}}\right)-x_{j h}^{i l \mathcal{T}-1}\right] \times\left[x_{j h}^{i l}-x_{j h}^{i l \mathcal{T}}\right] \\
+\sum_{j=1}^{J} \sum_{k=1}^{K} \sum_{h=1}^{H} \sum_{l=1}^{L}\left[y_{k h l}^{j \mathcal{T}}+\alpha\left(\frac{\partial r^{j}\left(\bar{x}^{\mathcal{T}}, \bar{y}^{\mathcal{T}}\right)}{\partial y_{k h l}^{j}}+\frac{\partial c_{k h l}^{j}\left(\bar{y}_{k h l}^{j \mathcal{T}}\right)}{\left.\partial y_{k h l}^{j}\right)}+\bar{\gamma}_{j}^{\mathcal{T}}+\hat{c}_{k h l}^{j}\left(\bar{y}^{\mathcal{T}}\right)-\bar{\rho}_{3 k h l}^{\mathcal{T}}\right)-y_{k h l}^{j \mathcal{T}-1}\right] \\
\times\left[y_{k h l}^{j}-y_{k h l}^{j \mathcal{T}}\right] \\
+\sum_{j=1}^{J}\left[\gamma_{j}^{\mathcal{T}}+\alpha\left(\sum_{i=1}^{I} \sum_{l=1}^{L} \sum_{h=1}^{H} \bar{x}_{j h}^{i l \mathcal{T}}-\sum_{k=1}^{K} \sum_{h=1}^{H} \sum_{l=1}^{L} \bar{y}_{k h l}^{j \mathcal{T}}\right)-\gamma_{j}^{\mathcal{T}-1}\right] \times\left[\gamma_{j}-\gamma_{j}^{\mathcal{T}}\right] \\
+\sum_{k=1}^{K} \sum_{h=1}^{H} \sum_{l=1}^{L}\left[\rho_{3 k h l}^{\mathcal{T}}+\alpha\left(\sum_{j=1}^{J} \bar{y}_{k h l}^{j \mathcal{T}}-d_{k h l}\left(\bar{\rho}_{3}^{\mathcal{T}}\right)\right)-\rho_{3 k h l}^{\mathcal{T}-1}\right] \times\left[\rho_{3 k h l}-\rho_{3 k h l}^{\mathcal{T}}\right] \geq 0, \\
\forall\left(x, y, \gamma, \rho_{3}\right) \in \mathcal{K} .
\end{gathered}
$$

\section{Step 3: C onvergence Verification}

If $\left|x_{j h}^{i l \mathcal{T}}-x_{j h}^{i l \mathcal{T}-1}\right| \leq \epsilon_{1}\left|y_{k h l}^{j \mathcal{T}}-y_{k h l}^{j \mathcal{T}-1}\right| \leq \epsilon_{1}\left|\gamma_{j}^{\mathcal{T}}-\gamma_{j}^{\mathcal{T}-1}\right| \leq \epsilon,\left|\rho_{3 k h l}^{\mathcal{T}}-\rho_{3 k h l}^{\mathcal{T}-1}\right| \leq \epsilon$, for all $i=1, \cdots, I$; $l=1, \ldots, L ; j=1, \cdots, J ; h=1, \ldots, H ; k=1, \cdots, K$, with $\epsilon>0$, a pre-specified tolerance, then stop; otherwise, set $\mathcal{T}:=\mathcal{T}+1$, and go to Step 1 .

Both variational inequality subproblems (33) and (34) can be solved explicitly and in closed form since they are actually quadratic programming problems and the feasible set is a Cartesian product consisting of the the product of $\mathcal{K}^{1}$ and $\mathcal{K}^{2}$ The former has a simple network structure, whereas the latter consists of the cross product of the nonnegative orthants: $R_{+}^{J K H L}, R_{+}^{J}$, and $R_{+}^{J K H L}$, and corresponding to the variables $x, y, \gamma$, and $\rho_{3}$, respectively. In fact, the subproblems in (33) and (34) corresponding to the $x$ variables can be solved using exact equilibration (cf. Dafermos and Sparrow (1969) and Nagurney (1999)), whereas the remainder of the variables in (33) and (34) can be obtained by explicit formulae.

We now, for completeness, and also to illustrate the simplicity of the proposed computational procedure in the context of the international financial network model, state the 
explicit formulae for the computation of the $\bar{y}^{\mathcal{T}}$, the $\bar{\gamma}^{\mathcal{T}}$, and the $\bar{\rho}_{3}^{\mathcal{T}}$ (cf. (33)). The $y^{\mathcal{T}}, \gamma^{\mathcal{T}}$, and $\rho_{3}^{\mathcal{T}}$ can then be computed for (34) in an analogous fashion.

\section{Computation of the Financial Products from the Intermediaries}

In particular, compute, at iteration $\mathcal{T}$, the $\bar{y}_{k h l}^{j \mathcal{T}} \mathrm{s}$, according to:

$$
\begin{gathered}
\bar{y}_{k h l}^{j \mathcal{T}}=\max \left\{0, \bar{y}_{k h l}^{j \mathcal{T}-1}-\alpha\left(\frac{\partial r^{j}\left(\bar{x}^{\mathcal{T}-1}, \bar{y}^{\mathcal{T}-1}\right)}{\partial y_{k h l}^{j}}+\frac{\partial c_{k h l}^{j}\left(\bar{y}_{k h l}^{j \mathcal{T}-1}\right)}{\partial y_{k h l}^{j}}+\bar{\gamma}_{j}^{\mathcal{T}-1}+\hat{c}_{k h l}^{j}\left(\bar{y}^{\mathcal{T}-1}\right)-\bar{\rho}_{3 k h l}^{\mathcal{T}-1}\right)\right\} \\
\forall j, k, h, l
\end{gathered}
$$

\section{Computation of the Prices}

At iteration $\mathcal{T}$, compute the $\bar{\gamma}_{j}^{\mathcal{T}} \mathrm{s}$ according to:

$$
\bar{\gamma}_{j}^{\mathcal{T}}=\max \left\{0, \bar{\gamma}_{j}^{\mathcal{T}-1}-\alpha\left(\sum_{i=1}^{I} \sum_{l=1}^{L} \sum_{h=1}^{H} \bar{x}_{j h}^{i l \mathcal{T}-1}-\sum_{k=1}^{K} \sum_{h=1}^{H} \sum_{l=1}^{L} \bar{y}_{k h l}^{j \mathcal{T}-1}\right)\right\}, \quad \forall j,
$$

whereas the $\bar{\rho}_{3 k h l}^{\mathcal{T}} \mathrm{s}$ are computed explicitly and in closed form according to:

$$
\bar{\rho}_{3 k h l}^{\mathcal{T}}=\max \left\{0, \bar{\rho}_{3 k h l}^{\mathcal{T}-1}-\alpha\left(\sum_{j=1}^{J} \bar{y}_{k h l}^{j \mathcal{T}-1}-d_{k h l}\left(\bar{\rho}_{3}^{\mathcal{T}-1}\right)\right)\right\}, \quad \forall k, h, l .
$$

In the next Section, we apply the modified projection method to solve several international financial network examples.

We now state the convergence result for the modified projection method for this model.

\section{Theorem 8: C onvergence}

Assume that the function that enters the variational inequality (20) (or (23)) has at least one solution and satisfies the conditions in Theorem 4 and in Theorem 7. Then the modified projection method described above converges to the solution of the variational inequality (20) or (23).

Proof: According to K orpelevich (1977), the modified projection method converges to the solution of the variational inequality problem of the form (20), provided that the function 
$F$ that enters the variational inequality is monotone and Lipschitz continuous and that a solution exists. Existence of a solution follows from Theorem 3, monotonicity follows Theorem 4, and Lipschitz continuity, in turn, follows from Theorem 7. The proof is complete.

Of course, the algorithm may converge even if the conditions in Theorems 4 and 7 do not hold in which case the algorithm, nevertheless, converges to the equilibrium solution. 


\section{N umerical Examples}

In this Section, we apply the modified projection method to several numerical examples. The modified projection method was implemented in FORTRAN and the computer system used was a DEC Alpha system located at the University of Massachusetts at Amherst. For the solution of the induced network subproblems in the $x$ variables we utilized the exact equilibration algorithm (see Dafermos and Sparrow (1969), Nagurney (1999), and the references therein). The other variables were determined in the computation and adaptation steps of the modified projection method explicitly and in closed form as described in the preceding section.

The convergence criterion used was that the absolute value of the flows and prices between two successive iterations differed by no more than $10^{-4}$. For the examples, $\alpha$ was set to .01 in the algorithm.

We assumed in all the examples that the risk functions were of the form (3) and (11), that is, that risk was represented through variance-covariance matrices for both the source agents in the countries and for the intermediaries.

We initialized the modified projection method as follows: We set $x_{j h}^{i l}=\frac{S^{i l}}{J H}$ for each source agent $i$ and country $l$. All the other variables, that is, the initial vectors $y, \gamma$, and $\rho_{3}$ were all set to zero.

We solved two sets of numerical examples, with three examples each. Detailed descriptions are given below.

\section{Example 1}

The first set of numerical examples consisted of one country, two source agents, two currencies, two intermediaries, and two financial products. Hence, $L=1, I=2, H=2$, $J=2$, and $K=2$, for this and the subsequent two numerical examples. The international financial network for the first three examples is depicted in Figure 2.

The data for the first example were constructed for easy interpretation purposes. The financial holdings of the two source agents were: $S^{11}=20$ and $S^{21}=20$. The variance- 


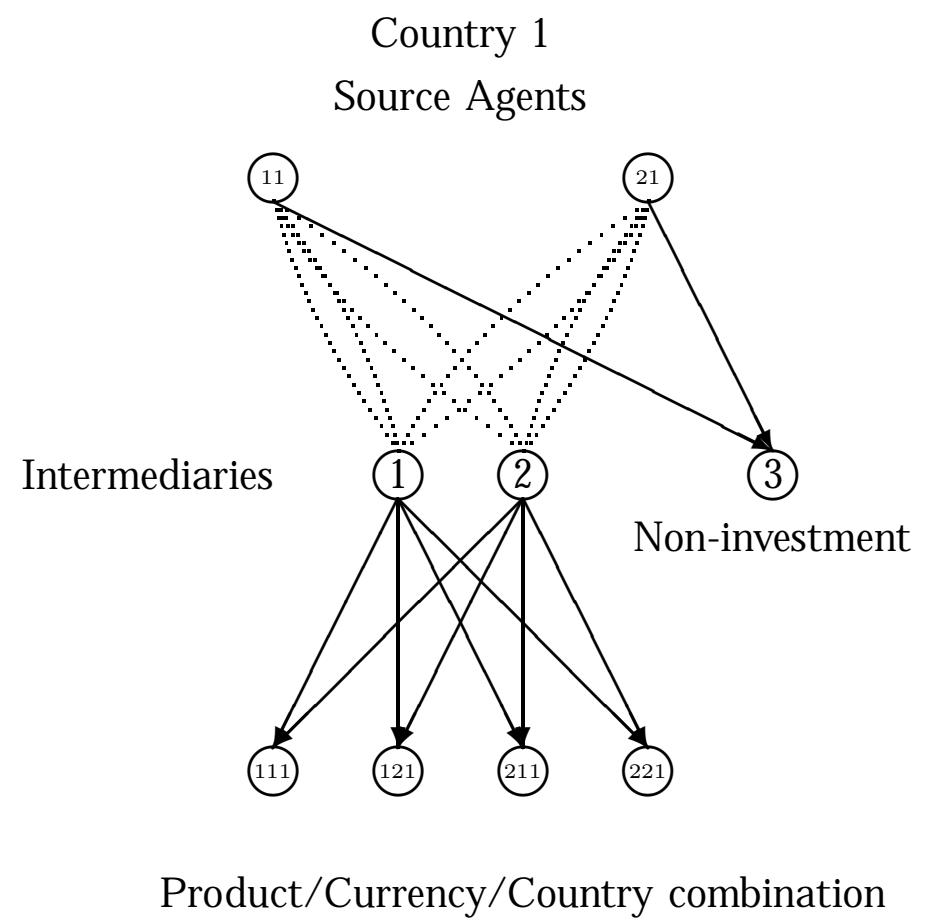

Figure 2: International Financial Network for the First Set of Numerical Examples 
covariance matrices $Q^{i l}$ and $Q^{j}$ were equal to the identity matrices (appropriately dimensioned) for all source agents and all intermediaries, respectively.

The transaction cost functions faced by the source agents associated with transacting with the intermediaries (cf. (1)) were given by:

$$
c_{j h}^{i l}\left(q_{j h}^{i l}\right)=.5\left(q_{j h}^{i l}\right)^{2}+3.5 q_{j h}^{i l}, \quad \text { for } i=1,2 ; l=1 ; j=1,2 ; h=1,2 .
$$

The handling costs of the intermediaries, in turn (see (7)), were given by:

$$
c_{j}(x)=.5\left(\sum_{i=1}^{2} \sum_{h=1}^{2} x_{j h}^{i 1}\right)^{2}, \quad \text { for } j=1,2 .
$$

The transaction costs of the intermediaries associated with transacting with the source agents were (cf. (8)) given by:

$$
\hat{c}_{j h}^{i l}\left(q_{j h}^{i l}\right)=1.5 q_{j h}^{i l}{ }^{2}+3 q_{j h}^{i l}, \quad \text { for } i=1,2 ; l=1 ; j=1,2 ; h=1,2 .
$$

The demand functions at the demand markets (refer to (16)) were:

$$
\begin{aligned}
& d_{111}\left(\rho_{3}\right)=-2 \rho_{3111}-1.5 \rho_{3121}+1000, \quad d_{121}\left(\rho_{3}\right)=-2 \rho_{3121}-1.5 \rho_{3111}+1000, \\
& d_{211}\left(\rho_{3}\right)=-2 \rho_{3211}-1.5 \rho_{3221}+1000, \quad d_{221}\left(\rho_{3}\right)=-2 \rho_{3221}-1.5 \rho_{3211}+1000 .
\end{aligned}
$$

and the transaction costs between the intermediaries and the consumers at the demand markets (see (11)) were given by:

$$
\hat{c}_{j k l}\left(q_{j k l}\right)=q_{j k l}+5, \quad \text { for } j=1,2 ; k=1,2 ; l=1 .
$$

We assumed for this and the subsequent examples that the transaction costs as perceived by the intermediaries and associated with transacting with the demand markets were all zero, that is, $c_{k h l}^{j}\left(x_{k h l}^{j}\right)=0$, for all $j, k, l$.

The modified projection method converged and yielded the following equilibrium financial flow pattern:

$$
x^{*}:=x_{11}^{11^{*}}=x_{12}^{11 *}=x_{21}^{11 *}=x_{22}^{11 *}=x_{11}^{21^{*}}=x_{22}^{21^{*}}=x_{21}^{21^{*}}=x_{22}^{21^{*}}=5.0000 ;
$$




$$
y^{*}:=y_{111}^{1 *}=y_{121}^{1 *}=y_{211}^{1 *}=y_{221}^{1 *}=y_{111}^{2 *}=y_{121}^{2 *}=y_{211}^{2 *}=y_{221}^{2 *}=5.0000 \text {. }
$$

The vector $\gamma^{*}$ had components: $\gamma_{1}^{*}=\gamma_{2}^{*}=262.8566$, and the computed demand prices at the demand markets were: $\rho_{3111}^{*}=\rho_{3121}^{*}=\rho_{3211}^{*}=\rho_{3221}^{*}=282.856$.

We also, for completeness, recover the equilibrium prices associated with the source agents according to the discussion following (23). In particular, we had that all components of the vector $\rho_{1}^{*}$ were identically equal to 214.8566 .

It is easy to verify that the optimality/ equilibrium conditions were satisfied with good accuracy. Note that in this example, constraint (5) was tight for both source agents, that is, there was zero flow on the links connecting node 3 with top tier nodes. Thus, it was optimal for both source agents to invest their entire financial holdings in the instrument made available by each of the two intermediaries in each of the two currencies. Clearly, due to the input data in this highly stylized example, the equilibrium financial flow pattern could have been "predicted" even without any computations; however, the same does not hold (even in this quite "symmetric") example for the prices.

\section{Example 2}

In the second example, we kept the data as in Example 1 except for the following changes: we increased the demand associated with the first product in the first currency so that the new demand functions were now given by:

$$
d_{111}\left(\rho_{3}\right)=-2 \rho_{3111}-1.5 \rho_{3121}+1010,
$$

with the demand functions for the second product (and second currency) remaining unchanged.

The modified projection method converged and yielded the following equilibrium financial flow pattern:

$$
\begin{gathered}
x^{*}:=x_{11}^{11^{*}}=x_{12}^{11^{*}}=x_{21}^{11 *}=x_{22}^{11^{*}}=x_{11}^{21^{*}}=x_{22}^{21 *}=x_{21}^{21 *}=x_{22}^{21^{*}}=5.0000 ; \\
y^{*}:=y_{111}^{1 *}=6.6288, y_{121}^{1 *}=3.7717, y_{211}^{1 *}=4.8003, y_{221}^{1 *}=4.8003, \\
y_{111}^{2 *}=6.6288, y_{121}^{2 *}=3.7717, y_{211}^{2 *}=4.8003, y_{221}^{2 *}=4.8003 .
\end{gathered}
$$


As was the case in Example 1, both source agents allocated the entirety of their funds to the instrument in the two currencies; thus, there was no non-investment.

The vector $\gamma^{*}$ had components: $\gamma_{1}^{*}=\gamma_{2}^{*}=914.9965$, and the computed demand prices at the demand markets were: $\rho_{3111}^{*}=979.9990, \rho_{3121}^{*}=0.0000, \rho_{3211}^{*}=285.7142, \rho_{3221}^{*}=$ 285.7142 .

Clearly, since the demand for the first product in the first country and currency increased, the financial flows between the two intermediaries and that demand market increased (as did the equilibrium price at that demand market), whereas the others decreased vis a vis the analogous ones in Example 1.

We also computed (as discussed in Example 1 and following (23)) the new equilibrium prices associated with the top tier of nodes in the international financial network and now the new equilibrium vector $\rho_{1}^{*}$ had all of its components equal to 215.5708 .

\section{Example 3}

In the third and final example in this set, we kept the data as in Example 2 except for the following changes: we increased the risk associated with source agent 1 in country 1 transacting with intermediary 1 and 2 in currency 1 . Hence, rather than having the first source agent's variance-covariance matrix now being equal to the identity matrix, the first two diagonal elements of its matrix were now set to 2 .

The modified projection method converged and yielded the following new equilibrium financial flow pattern:

$$
\begin{gathered}
x^{*}:=x_{11}^{11^{*}}=4.2857, x_{12}^{11^{*}}=5.7143, x_{21}^{11^{*}}=4.2857, x_{22}^{11^{*}}=5.7143, \\
x_{11}^{21 *}=x_{22}^{21^{*}}=x_{21}^{21 *}=x_{22}^{21^{*}}=5.0000 .
\end{gathered}
$$

A gain, there was no non-investment.

The vectors: $y^{*}, \gamma^{*}$, and $\rho_{3}^{*}$ remained identical to those obtained in Example 2. Thus, as expected, since the risk associated with the first currency increased, as perceived by the first source agent, he reallocated his financial funds so that a greater volume was now placed in the second currency. 


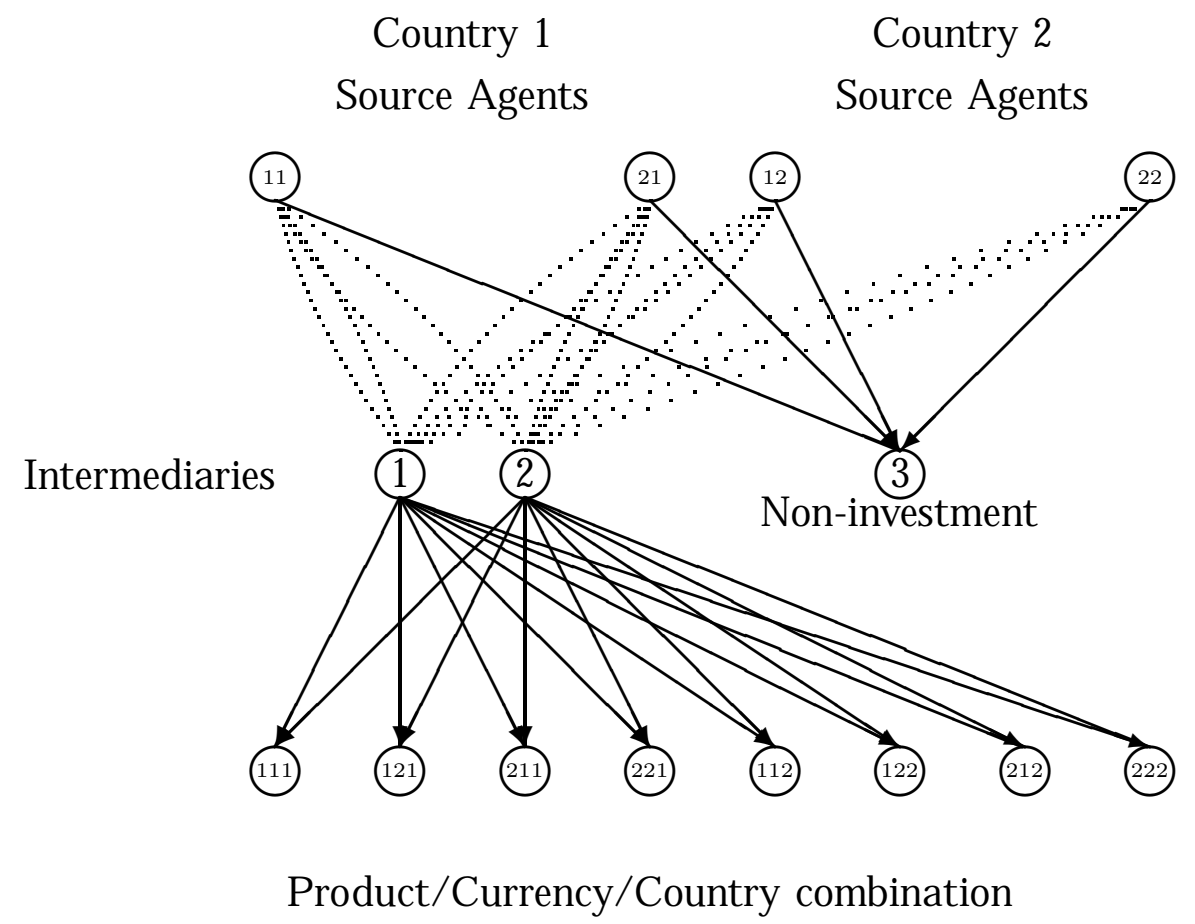

Figure 3: International Financial Network for the Second Set of Numerical Examples

The equilibrium prices associated with the source agents remained as those in Example 2 except that now the prices associated with the first source agent were now all equal to 210.5709 .

\section{Example 4}

In the second set of numerical examples, the internationaal financial network was as given in Figure 3. These three examples consisted of two countries with two source agents in each country; two currencies, two intermediaries, and two financial products. Hence, $L=2$, $I=2, H=2, J=2$, and $K=2$.

The data for the first example in this set was constructed for easy interpretation purposes and to create a baseline from which the simulations could be conducted. In fact, we essentially "replicated" the data for the first country as it appeared in Example 1 in order to construct the data for the second country. 
Specifically, the financial holdings of the source agents were: $S^{11}=20, S^{21}=20, S^{12}=20$, and $S^{22}=20$. The variance-covariance matrices $Q^{i l}$ and $Q^{j}$ were equal to the identity matrices (appropriately dimensioned) for all source agents in each country and for all intermediaries, respectively.

The transaction cost functions faced by the source agents associated with transacting with the intermediaries were given by:

$$
c_{j h}^{i l}\left(q_{j h}^{i l}\right)=.5\left(q_{j h}^{i l}\right)^{2}+3.5 q_{j h}^{i l}, \quad \text { for } i=1,2 ; l=1,2 ; j=1,2 ; h=1,2 .
$$

The handling costs of the intermediaries (since the number of intermediaries in this set is still equal to two) remained as in Example 1, that is, they were given by:

$$
c_{j}(x)=.5\left(\sum_{i=1}^{2} \sum_{h=1}^{2} x_{j h}^{i 1}\right)^{2}, \quad \text { for } j=1,2 .
$$

The transaction costs of the intermediaries associated with transacting with the source agents in the two countries were given by:

$$
\hat{c}_{j h}^{i l}\left(q_{j h}^{i l}\right)=1.5 q_{j h}^{i l}{ }^{2}+3 q_{j h}^{i l}, \quad \text { for } i=1,2 ; l=1,2 ; j=1,2 ; h=1,2 .
$$

The demand functions at the demand markets were:

$$
\begin{array}{ll}
d_{111}\left(\rho_{3}\right)=-2 \rho_{3111}-1.5 \rho_{3121}+1000, & d_{121}\left(\rho_{3}\right)=-2 \rho_{3121}-1.5 \rho_{3111}+1000, \\
d_{211}\left(\rho_{3}\right)=-2 \rho_{3211}-1.5 \rho_{3221}+1000, & d_{221}\left(\rho_{3}\right)=-2 \rho_{3221}-1.5 \rho_{3211}+1000, \\
d_{112}\left(\rho_{3}\right)=-2 \rho_{3112}-1.5 \rho_{3122}+1000, & d_{122}\left(\rho_{3}\right)=-2 \rho_{3122}-1.5 \rho_{3112}+1000, \\
d_{212}\left(\rho_{3}\right)=-2 \rho_{3212}-1.5 \rho_{3222}+1000, & d_{222}\left(\rho_{3}\right)=-2 \rho_{3222}-1.5 \rho_{3212}+1000,
\end{array}
$$

and the transaction costs between the intermediaries and the consumers at the demand markets were given by:

$$
\hat{c}_{j k l}\left(q_{j k l}\right)=q_{j k l}+5, \quad \text { for } j=1,2 ; k=1,2 ; l=1,2 .
$$


The modified projection method converged and yielded the following equilibrium financial flow pattern:

$$
\begin{gathered}
x^{*}:=x_{11}^{11^{*}}=x_{12}^{11 *}=x_{21}^{11 *}=x_{22}^{11 *}=x_{11}^{21 *}=x_{22}^{21 *}=x_{21}^{21 *}=x_{22}^{21 *}=5.0000, \\
x_{11}^{12 *}=x_{12}^{12 *}=x_{21}^{12 *}=x_{22}^{12 *}=x_{11}^{22 *}=x_{22}^{22 *}=x_{22}^{21 *}=x_{22}^{22 *}=5.0000 \\
y^{*}:=y_{111}^{1 *}=y_{121}^{1 *}=y_{211}^{1 *}=y_{221}^{1 *}=y_{111}^{2 *}=y_{121}^{2 *}=y_{211}^{2 *}=y_{221}^{2 *}=5.0000, \\
y_{112}^{1 *}=y_{122}^{1 *}=y_{212}^{1 *}=y_{222}^{1 *}=y_{112}^{2 *}=y_{122}^{2 *}=y_{212}^{2 *}=y_{222}^{2 *}=5.0000 .
\end{gathered}
$$

The vector $\gamma^{*}$ had components: $\gamma_{1}^{*}=\gamma_{2}^{*}=262.8486$, and the computed demand prices at the demand markets were: $\rho_{3111}^{*}=\rho_{3121}^{*}=\rho_{3211}^{*}=\rho_{3221}^{*} \rho_{3112}^{*}=\rho_{3122}^{*}=\rho_{3212}^{*}=\rho_{3222}^{*}=$ 282.8591.

The components of the vector $\rho_{1}^{*}$ were identically equal to 194.8486 .

\section{Example 5}

Example 5 was constructed from the preceding example as follows. We kept the data as in Example 4 except that we changed the demand functions associated with the second country so that:

$$
\begin{aligned}
& d_{112}\left(\rho_{3}\right)=-2 \rho_{3112}-1.5 \rho_{3122}+1010, \quad d_{122}\left(\rho_{3}\right)=-2 \rho_{3122}-1.5 \rho_{3112}+1020, \\
& d_{212}\left(\rho_{3}\right)=-2 \rho_{3212}-1.5 \rho_{3222}+1030, \quad d_{222}\left(\rho_{3}\right)=-2 \rho_{3222}-1.5 \rho_{3212}+1040 .
\end{aligned}
$$

The modified projection method converged and yielded the following equilibrium financial flow pattern:

$$
\begin{gathered}
x^{*}:=x_{11}^{11 *}=x_{12}^{11 *}=x_{21}^{11 *}=x_{22}^{11 *}=x_{11}^{21 *}=x_{22}^{21 *}=x_{21}^{21 *}=x_{22}^{21 *}= \\
x_{11}^{12 *}=x_{12}^{12 *}=x_{21}^{12 *}=x_{22}^{12^{*}}=x_{11}^{22 *}=x_{22}^{22 *}=x_{22}^{21 *}=x_{22}^{22 *}=5.0000 \\
y^{*}:=y_{111}^{1 *}=y_{121}^{1 *}=y_{211}^{1 *}=y_{221}^{1 *}=3.9999, \\
y_{112}^{1 *}=3.7712, y_{122}^{1 *}=6.6286, y_{212}^{1 *}=5.3712, y_{22}^{1 *}=8.2286, \\
y_{111}^{2 *}=y_{121}^{2 *}=y_{211}^{2 *}=y_{221}^{2 *}=3.999,
\end{gathered}
$$




$$
y_{112}^{2 *}=3.7712, y_{122}^{2 *}=6.6286, y_{212}^{2 *}=5.3712, y_{222}^{2 *}=8.2286
$$

The vector $\gamma^{*}$ had components: $\gamma_{1}^{*}=\gamma_{2}^{*}=266.4286$, and the computed demand prices at the demand markets were: $\rho_{3111}^{*}=\rho_{3121}^{*}=\rho_{3211}^{*}=\rho_{3221}^{*}=283.4285 \rho_{3112}^{*}=282.7425, \rho_{3122}^{*}=$ 291.3144, $\rho_{3212}^{*}=287.5425, \rho_{3222}^{*}=296.144$.

The components of the vector $\rho_{1}^{*}$ were identically equal to 198.4286 .

Hence, in this example, vis a vis the solution of Example 4, the financial flows between the intermediaries and the demand markets were reallocated to satisfy the increased demand.

\section{Example 6}

Example 6 was constructed from the preceding example as follows. We kept the data as in Example 5 except that we increased the first diagonal element of the variance-covariance matrix for the first source agent in the first country from 1 to 2 .

The modified projection method converged and yielded the following equilibrium financial flow pattern: all the components of the vector $x^{*}$ corresponding to the flow into intermediary 1 were equal to 4.0476 whereas all those into intermediary 2 were equal to 5.9524 .

$$
\begin{gathered}
y^{*}:=y_{111}^{1 *}=y_{121}^{1 *}=y_{211}^{1 *}=y_{221}^{1 *}=3.0475 \\
y_{112}^{1 *}=2.8188, y_{122}^{1 *}=5.6762, y_{212}^{1 *}=4.4188, y_{222}^{1 *}=7.2672 \\
y_{111}^{2 *}=y_{121}^{2 *}=y_{211}^{2 *}=y_{221}^{2 *}=4.9523 \\
y_{112}^{2 *}=4.7236, y_{122}^{2 *}=7.5809, y_{212}^{2 *}=6.3236, y_{222}^{2 *}=9.1809
\end{gathered}
$$

The vector $\gamma^{*}$ had components: $\gamma_{1}^{*}=269.2857, \gamma_{2}^{*}=263.5715$, and the computed demand prices at the demand markets remained as in the solution of Example 5.

These examples have been presented to show both the model and the computational procedure. Obviously, different input data and dimensions of the problems solved will affect the equilibrium financial flow and price patterns. One now has a powerful tool with which to explore the effects of perturbations to the data as well as the effects of changes in the number of source agents, countries, currencies, and/ or products. 


\section{Summary and Conclusions}

In this paper, we have developed a framework for the modeling, analysis, and computation of solutions to international financial problems with intermediation in the context of the medium of networks. In particular, we have proposed an international financial network model consisting of three tiers of decision-makers: the source agents, the financial intermediaries, and the consumers associated with the demand markets for distinct financial products in distinct currencies and countries. We modeled the behavior of the decision-makers, derived the optimality conditions as well as the governing equilibrium conditions which reflect (possible) competition among decision-makers at the same tier of nodes but cooperation between tiers of nodes. The framework allows for the handling of as many countries, as many source agents in each country, as many currencies in which the financial products can be obtained, and as many financial intermediaries, as mandated by the specific application.

The formulation of the equilibrium conditions was shown to be equivalent to a finitedimensional variational inequality problem. The variational inequality problem was then utilized to obtain qualitative properties of the equilibrium financial flow and price pattern as well as to propose a computational procedure for the numerical determination of the equilibrium flows in particular examples. The algorithm was subsequently applied to several international financial network examples to illustrate both the model as well as the computational procedure.

This framework generalizes the recent work of Nagurney and Ke (2001) in financial networks with intermediation to the international dimension. 


\section{A cknowledgments}

This research was supported, in part, by NSF Grant No.: IIS-0002647 and, in part, by NSF Grant N o.: CM S- 0085720 as well as by an AT \&T Industrial Ecology Fellowship. This support is gratefully acknowledged. 


\section{R eferences}

B azaraa, M. S., Sherali, H. D., and Shetty, C. M. (1993). "Nonlinear Programming: Theory and Algorithms," J ohn W iley \& Sons, New York.

Bertsekas, D. P., and Tsitsiklis, J. N. (1989). "Parallel and Distributed Computation Numerical M ethods," Prentice Hall, Englewood Cliffs, New J ersey.

B oard of G overnors (1980). "Introduction to F low of Funds," Flow of Funds Section, Division of Research and Statistics, Federal Reserve System, Washington, DC, J une.

Charnes, A., and Cooper, W. W . (1967). Some network characterizations for mathematical programming and accounting approaches to planning and control, The Accounting Review 42, 24-52.

Cohen, J. (1987). "The Flow of Funds in Theory and Practice," Financial and M onetary Studies 15, K luwer A cademic Publishers, Dordrecht, The N etherlands.

Dafermos, S., and Nagurney, A . (1987). Oligopolistic and competitive behavior of spatially separated markets, Regional Science and Urban Economics 17, 245-254.

Dafermos, S., and Sparrow, F. T. (1969). The traffic assignment problem for a general network, J ournal of Research of the National Bureau of Standards 73B, 91-118.

Gabay, D., and Moulin, H. (1980). On the uniqueness and stability of Nash equilibria in noncooperative games, A. B ensoussan, P. K leindorfer, and C. S. Tapiero, editors, "A pplied Stochastic Control of E conometrics and M anagement Science," North-Holland. A msterdam, The Netherlands.

K inderlehrer, D., and Stampacchia, G. (1980). "A n Introduction to Variational Inequalities and Their Application," A cademic Press, New York.

K orpelevich, G. M. (1977). The extragradient method for finding saddle points and other problems, Matekon 13, 35-49.

Markowitz, H. M. (1959). "Portfolio Selection: Efficient Diversification of Investments," 
J ohn Wiley \& Sons, Inc., New York.

Nagurney, A . (1999). "Network E conomics: A Variational Inequality A pproach," second and revised edition, K luwer A cademic Publishers, Dordrecht, The Netherlands.

Nagurney, A . (2001). F inance and variational inequalities, Quantitative Finance 1, 309-317. Nagurney, A., Dong, J ., and Hughes, M. (1992). Formulation and computation of general financial equilibrium, Optimization 26, 339-354.

Nagurney, A., and Hughes, M. (1992). Financial flow of funds networks, Networks 2, 145161.

Nagurney, A., and Ke, K. (2001). Financial networks with intermediation, Quantitative Finance 1, 441-451.

Nagurney, A., and Siokos, S. (1997). "F inancial Networks: Statics and Dynamics," SpringerVerlag, Heidelberg, Germany.

Nagurney, A., and Zhao, L. (1993). Networks and variational inequalities in the formulation and computation of market disequilibria: the case of direct demand functions, Transportation Science 27, 4-15.

Nash, J. F. (1950). Equilibrium points in n-person games, in Proceedings of the National Academy of Sciences, USA 36, 48-49.

Nash, J . F . (1951). Noncooperative games, Annals of Mathematics 54, 286-298.

Quesnay, F. (1758). "Tableau Economique," reproduced in facsimile with an introduction by H. Higgs by the B ritish E conomic Society, 1895.

Storoy, S., Thore, S., and B oyer, M. (1975). Equilibrium in linear capital market networks, The J ournal of Finance 30, 1197-1211.

Thore, S. (1969). Credit networks, Economica 36, 42-57. 
Thore, S. (1980). "Programming the Network of Financial Intermediation," Universitetsforslaget, Oslo, Norway. 